\title{
Pueblos indígenas y minorías nacionales: similitudes y diferencias en la protección internacional de sus derechos
}

Indigenous peoples and national minorities: similarities and differences in the international protection of their rights

María José Andrade

Universidad Pública de Navarra, España

Artículo de investigación

Fecha de recepción: 29 de septiembre de 2017

Fecha de aceptación: 16 de abril de 2018

\section{Para citar este artículo}

Andrade, M. J. (2018). Pueblos indígenas y minorías nacionales: similitudes y diferencias en la protección internacional de sus derechos. Revista Campos en Ciencias Sociales, 6(2), 13-48. Bogotá D.C, Colombia: Universidad Santo Tomás. DOI: https://doi.org/10.15332/s2339-3688.2018.0002.01

1 Doctora (c) en Derecho. Correo electrónico: mandradem@alumni.unav.es 


\title{
RESUMEN
}

Pueblos indígenas y minorías nacionales son grupos étnicos con características comunes, pero también, con diferencias relevantes. Este artículo examina los instrumentos que protegen sus derechos en la comunidad internacional y compara el contenido de los mismos, planteando que la diferencia más significativa es el reconocimiento del derecho de autodeterminación a los pueblos indígenas - no así a las minorías nacionales-, del cual derivan otros relevantes. Por otro lado, a pesar de los avances en la codificación internacional de sus derechos, ambos comparten falencias en su aplicación debido a la reticencia de los Estados a dar atribuciones a estos colectivos. La comparación entre ambos grupos puede aportar en buena medida en las contribuciones mutuas que puedan hacerse en el ámbito de la protección de sus derechos.

Palabras clave: pueblos indígenas, minorías culturales, derechos humanos, grupos étnicos.

\begin{abstract}
Indigenous peoples and national minorities are ethnic groups with common features but also relevant differences. This article studies the instruments that protect their rights in the international community and compares their contents. The text states that the main difference is the recognition of the right to self-determination of indigenous peoples -and not of national minorities-, from which other important rights derive. On the other side, despite the progresses in the international codification of their rights, both groups share the lack of implementation and control mechanisms, due to the States' reluctance to give attributions to these groups. The comparison between both groups can be useful for the mutual contributions that can be made in the field of the protection of their rights.
\end{abstract}

Keywords: Indigenous peoples, cultural minorities, human rights, ethnic groups. 


\section{INTRODUCCIÓN}

Pueblos indígenas y minorías nacionales son grupos étnicos con importantes características en común pero también con diferencias relevantes, lo que ha llevado a que la protección internacional de sus derechos haya tomado caminos distintos. A través de la revisión bibliográfica, del análisis de los instrumentos internacionales vigentes que protegen a estos colectivos y de la revisión de jurisprudencia pertinente, se sigue aquí una metodología comparada para presentar las similitudes y distinciones entre ellos y sus derechos reconocidos. Lo anterior, con el objetivo de aportar a las contribuciones que puedan hacerse mutuamente la protección de pueblos originarios y de minorías nacionales.

Ningún instrumento internacional establece una definición de aceptación universal o regional de minorías nacionales ni de pueblos indígenas. Esto, porque los Estados son reacios a reconocer derechos a estos grupos y los definen de maneras que no amenacen su soberanía. Por ejemplo, Alemania entiende que "minorías nacionales" son "grupos étnicos cuyos miembros son ciudadanos alemanes que viven en áreas de asentamiento bien definidas por un largo período de tiempo", lo que excluiría a los romaníes, que viven dispersos por el país (Tsekos, 2002, p. 27).

Con respecto a las minorías nacionales, hay consenso sobre sus características; a partir de él, Björn Arp (2008) las define como:

Un grupo de personas, caracterizadas por una identidad común que no es la constitutiva de su Estado de asentamiento, que tienen por regla general la ciudadanía de este Estado y que en cualquier caso han estado asentados en él desde hace mucho tiempo, de manera que se trata de una comunidad autóctona en ese Estado (p. 405).

Capotorti añade su inferioridad numérica en relación con el resto de la población, su posición no dominante y su voluntad de preservar su cultura. La definición del Relator Especial de la Subcomisión de Prevención de Discriminaciones y Protección de las Minorías de la ONU, es la siguiente: "un grupo numéricamente inferior al 
resto de la población de un Estado, en una posición no dominante, cuyos miembros -siendo nacionales del Estado- tienen características étnicas, religiosas o lingüísticas que difieren de las del resto de la población y muestran, aunque solo implícitamente, un sentido de solidaridad, dirigido hacia la preservación de su cultura, tradiciones, religión o lengua”2 (Capotorti, 1979, párr. 568). (Traducción propia).

Con base en los anteriores autores, en este artículo se entiende por minoría nacional a un grupo no dominante y numéricamente inferior al resto de la población de un Estado en el que están asentados desde hace mucho tiempo, integrado por personas que comparten una identidad cultural distinta de la mayoritaria -etnia, religión, tradiciones o costumbres-, la cual pretenden preservar.

Sin embargo, la protección de minorías nacionales sigue un concepto "minimalista" para efectos del derecho internacional (Pentassuglia, 2002, pp. 55-71), y así los Estados pueden negar su existencia en su territorio. Ejemplo de esto son Francia, Grecia y Turquía.

Tampoco hay una definición generalizada sobre pueblos indígenas. En 1989, un grupo de expertos bajo la dirección de la Unesco se reunió y estableció siete rasgos esenciales que describen a un pueblo: tradición histórica común, identidad racial o étnica, homogeneidad cultural, unidad lingüística, afinidad religiosa o ideológica, conexión territorial, y vida económica común. Un pueblo sería un grupo de individuos con algunas o todas las características enumeradas (Unesco, 1990). Además, estableció un criterio numérico (no requiere ser muy grande, pero sí mayor que una simple asociación dentro de un Estado) y señaló que debe tener la voluntad de ser identificado como pueblo o la consciencia de serlo, y posiblemente medios para manifestar esas características.

2 "A group numerically inferior to the rest of the population of a State, in a non-dominant position, whose members - being nationals of the State- possess ethnic, religious or linguistic characteristics differing from those of the rest of the population and show, if only implicitly, a sense of solidarity, directed towards preserving their culture, traditions, religion or language" (versión original en inglés). 
El término genera recelo en los Estados por sus repercusiones políticas y jurídicas: un pueblo tiene derecho a la autodeterminación, una de las principales demandas de las comunidades indígenas. Por lo tanto, "[n]o es una discusión teórica o científica, sino política” (Sobero y Calduch, 2006, p. 19).

Acerca del significado de indigena, Stavenhagen (1992) afirma que estos son "los descendientes de los pueblos que ocupaban un territorio dado cuando este fue invadido, conquistado o colonizado por una potencia o una población extranjera" (p. 128). Desde un punto de vista semántico, un elemento esencial de lo indígena es la prioridad temporal. La palabra proviene del latín indigenae (personas nacidas en un lugar determinado, frente a los advenae, que venían de otro); y autóctono del griego (grupo que existió primero en un lugar). Ambos términos se han utilizado tradicionalmente en el derecho internacional en inglés -indigenous- y francés autochtone- (Daes, 1996, párr. 10), y distinguen entre personas originarias de un país e inmigrantes o colonos (Gómez del Prado, 2002, p. 29).

La definición de pueblos indígenas, sin embargo, se deja a criterio de cada Estado, por lo que varían (incluso dentro de una región puede haber diferencias significativas). Además, hay propuestas doctrinales, indígenas -que establecen guías mínimas amplias para delimitar el concepto y abogan por su derecho ilimitado a autodefinirse-, y de organizaciones internacionales (Aguilar, 2007, pp. 327-355).

Los especialistas coinciden en un grupo de características. Primero, la continuidad histórica, que es la conservación por un tiempo prolongado hasta el presente, de uno o más de los siguientes elementos: ocupación de las tierras ancestrales, ascendencia común con sus habitantes originales, manifestaciones culturales determinadas (idioma, religión, sistema tribal, vestimenta, medios y estilos de vida, etc.), y residencia en ciertos lugares del país o del mundo (Martínez Cobo, 1987, párr. 380). Como para considerarse un pueblo indígena debe haber uno o más de estos elementos, se incluyen las comunidades que perdieron sus tierras ancestrales pero mantienen otros aspectos.

En segundo lugar está la marginación, explotación y pobreza, como descripción de su situación socioeconómica. Una tercera característica es su economía de supervivencia, 
generalmente de autoabastecimiento o primaria. Además, tienen una cosmovisión distinta de la del resto de la población, que incluye un vínculo espiritual con la tierra. Finalmente, debe considerarse la autoidentificación de los pueblos indígenas en los elementos subjetivos para definirlos, junto con el sentimiento común de pertenencia y el deseo de continuar perteneciendo a su grupo (Gómez del Prado, 2002, p. 14).

Por todo lo anterior, en este artículo se entiende que un pueblo indígena es un grupo no dominante, integrado por personas que se autoidentifican como indígenas y que comparten una identidad cultural distinta de la dominante, la que pretenden conservar y en la que es esencial su especial relación con el territorio que ocupan o que quieren ocupar, debido a que se relacionan ancestralmente con sus habitantes originales. Tienen una economía de supervivencia y una organización comunitaria, y actualmente se encuentran en situación de pobreza y marginación.

Algunos consideran a las comunidades indígenas como un tipo de minoría. Para Kymlicka (1996), los grupos indígenas de América son derechamente minorías nacionales que antes de ser incorporadas a los nuevos Estados tuvieron distintas formas de autogobierno, lo cual siguen demandando en la actualidad (pp. 2526). Pierre George (1985) también entiende a ambos grupos como íntimamente relacionados, pues, por ejemplo, incluye a los indios de América del Norte dentro de la categoría de "minorías históricas" (pp. 34-39). La línea divisoria entre ambos es delgada y su protección responde a derechos humanos de grupos subordinados y marginados (Stavenhagen, 1992, pp. 141-142). La separación se establece en la Declaración de Viena de 1993: el artículo 19 habla de minorías en general y el 20 se dedica a los pueblos originarios. Esto, porque aunque tienen atributos en común, también tienen diferencias relevantes.

Primero, "minoría" implica ser un grupo numéricamente inferior dentro de la sociedad, lo que no siempre es así en los pueblos indígenas: algunos son mayoría o parte importante de la población. 
Por otro lado, para los pueblos originarios es esencial la ocupación ancestral de las tierras que habitan y su especial vinculación con ellas, algo que no es necesariamente relevante para las minorías (de hecho, hay algunas que están dispersas).

En tercer lugar, las minorías nacionales están familiarizadas con ideas liberales y democráticas, mientras los indígenas siguen organizados según etnicidad o tradición. Así, cosas como el derecho de propiedad, el sistema judicial o el tributario, son elementos del Estado moderno "incompatibles con el modo de vida tradicional de muchos pueblos indígenas" (Eriksen, 2002, p. 130). Lo mencionado, porque las minorías han sido parte del proceso de modernización y se han beneficiado de él, mientras la mayoría de los indígenas se mantiene fuera de la modernidad, en situación de pobreza.

Por otra parte, las minorías nacionales son culturalmente homogéneas, lo que ha facilitado su movilización en torno a su identidad, esto esencialmente en Europa, región donde históricamente las minorías nacionales han sido tema de preocupación y conflicto, razón por la cual también ha realizado los desarrollos jurídicos más relevantes en la protección de sus derechos (Convenio Marco para la Protección de las Minorías Nacionales y Carta Europea para las Lenguas Regionales y Minoritarias, por ejemplo). Los pueblos indígenas son heterogéneos y están dispersos y fragmentados, dificultándose el impacto nacional de sus movilizaciones.

La práctica internacional ha tendido a tratar a ambos grupos como categorías distintas pero solapadas, por lo que comparten ciertas consideraciones normativas, especialmente en cuanto a no discriminación e integridad cultural. Empero, el movimiento indígena prefiere distanciarse de las minorías nacionales, para demandar un régimen distinto que les reconozca derechos más amplios, como los de la autodeterminación y la restitución de tierras. De hecho, cuando comenzaron a ser distinguidos, la posición de los pueblos indígenas empezó a ganar apoyo en la arena política y en acuerdos internacionales en los años noventa (Sarivaara, Maatta y Uusiautti, 2013, p. 370). 


\section{Protección internacional de las minorías nacionales}

La protección de minorías no se menciona en la Declaración Universal de Derechos Humanos de 1948, pero esta reconoce derechos que les afectan: la no discriminación (art. 2), la igualdad ante la ley (art. 7), la libertad de pensamiento, conciencia y religión (art. 18), la de opinión y expresión (art. 19), el derecho a la asamblea pacífica y la asociación (art. 20), el derecho a la educación (art. 26), y el derecho a participar libremente en la vida cultural de la comunidad (art. 27). De todos modos, no obliga a los Estados a adoptar medidas concretas para protegerles.

La Convención sobre Prevención y Sanción del Genocidio de 1948 es el primer tratado general que se refiere a la protección de minorías tras la Segunda Guerra Mundial de modo más directo: como víctimas de actos genocidas, "perpetrados con la intención de destruir, total o parcialmente, a un grupo nacional, étnico, racial o religioso, como tal" (art. 2). Aunque son contra individuos, se dirigen contra el grupo como entidad (Lemkin, 1944). De todas formas, solo garantiza su existencia física, omitiendo el "genocidio cultural", prohíbe los actos con la intención de destruir grupos, no los que pretenden prevenir su preservación o desarrollo, ignorando las amenazas más sutiles que pueden afectar a las minorías. El esfuerzo por asimilarlas puede tener el mismo resultado que su destrucción física, pues implica la muerte de una cultura.

El Pacto Internacional de Derechos Civiles y Políticos (PIDCP) de 1966 quiso reconocer ciertos derechos especiales a los miembros de minorías, afirmando:

En los Estados en que existan minorías étnicas, religiosas o lingüísticas, no se negará a las personas que pertenezcan a dichas minorías el derecho que les corresponde, en común con los demás miembros de su grupo, a tener su

3 En los borradores se barajó esta posibilidad, entendiendo por genocidio cultural la destrucción de características específicas del grupo por: a) traslado forzado de nińos a otro grupo humano, b) exilio forzado y sistemático de individuos representantes de la cultura de un grupo, c) prohibición del uso de la lengua nacional incluso en el ámbito privado, d) destrucción sistemática de libros impresos en lengua nacional o de trabajos religiosos o prohibición de nuevas publicaciones y, e) destrucción sistemática de monumentos históricos o religiosos o su desviación para otros usos, destrucción o dispersión de documentos y objetos de valor histórico, artístico o religioso, y de objetos usados en culto religioso (Thornberry, 1991, p. 71). 
propia vida cultural, a profesar y practicar su propia religión y a emplear su propio idioma (art. 27).

Por lo tanto, las minorías tienen derecho a tener su propia vida cultural, profesar y practicar su religión y usar su idioma; tres aspectos esenciales para la protección de minorías y su identidad particular, más allá de la existencia física. Por primera vez un instrumento jurídico de alcance universal obliga a los Estados a proteger específicamente los derechos de los miembros de las minorías. Ahora bien, el pacto limita la protección solo a las minorías "que existen”, deben ser grupos bien establecidos y el Estado debe reconocer su existencia antes de asegurar sus derechos.

Se ha criticado también que menciona a las minorías "étnicas, religiosas y lingüísticas", omitiendo las nacionales, raciales y culturales. Algunas categorías se solapan, pero la enumeración de los grupos protegidos debería cubrir todos los tipos (Roth, 1992, p. 90). Por otro lado, sigue una fórmula negativa, señalando que los derechos "no se negarán”, no establece obligaciones positivas al Estado sino solo su no interferencia. Esto contrasta con el resto del PIDCP, que tiene un tono positivo y dice que todos tienen determinados derechos. De todos modos, una interpretación restrictiva haría inútil el artículo 27, pues si no se le da un contenido más fuerte, realmente no añade nada al PIDCP. La lengua es motivo de no discriminación en varios instrumentos internacionales, y los demás derechos enunciados están protegidos en otros artículos del pacto, dirigidos a todas las personas - la libertad de pensamiento, conciencia y religión, en el artículo 18, y la de expresión, en el 19-. El principio de efectividad también respalda una interpretación positiva, los derechos enunciados son difíciles de ejercer sin acciones del Estado que los favorezcan. El texto del artículo 27 es al menos ambiguo y puede prestarse para interpretaciones muy distintas.

En general, el pacto es un instrumento de enorme trascendencia, pues plantea que la protección de minorías no se agota en preservar su existencia física, y que debe basarse en dos derechos universales: el derecho a no ser discriminado y el derecho a la identidad cultural.

El único instrumento de aplicación universal dedicado específicamente a las minorías nacionales es la Declaración de las Naciones Unidas sobre Derechos de Personas 
Pertenecientes a Minorías Nacionales o Étnicas, Religiosas y Lingüísticas de 1992, que mantiene el enfoque individualista, pero valora la dimensión colectiva de manera más directa que el PIDCP: el grupo no tiene derechos, pero reconoce una dimensión colectiva limitada, en una via media entre los derechos individuales y los derechos colectivos plenos (Thornberry, 1995, p. 54). Además, muestra una tendencia hacia la acción positiva del Estado, aunque en un lenguaje vago que incluye frases limitantes como "siempre que sea posible" o "cuando sea apropiado". La declaración es un paso adelante pero tímido -y no vinculante-, pues como mucho establece un estándar internacional mínimo con requerimientos limitados para los Estados. No obstante, su ámbito de aplicación es global.

Las minorías nacionales también pueden valerse de instrumentos universales dedicados a otros asuntos, pero que les afectan de manera indirecta. Por ejemplo, la Convención de la Unesco sobre la Lucha contra las Discriminaciones en la Esfera de la Enseñanza de 1960, cuyo artículo 1 define la discriminación hacia grupos y no solo personas, y su artículo 5 reconoce el derecho de las minorías nacionales a llevar a cabo sus actividades educacionales, incluyendo establecer instituciones o sistemas educacionales separados y el uso o la enseñanza de su propia lengua; la Convención sobre la Eliminación de todas las Formas de Discriminación Racial de 1965, que en su artículo 4 da un mandato para grupos basado en la no discriminación. No se dirige a grupos ni menciona a las minorías, pero autoriza medidas especiales necesarias para el fomento de grupos raciales o étnicos; la Declaración sobre Raza y Prejuicio Racial de la Unesco de 1978, que trata aspectos relacionados con el estatus de grupo y minoría, enfatizando la protección de la identidad y el desarrollo de los grupos, prohibiendo la asimilación forzada y recalcando la necesidad de acción positiva en estos asuntos; y la Declaración sobre la Eliminación de Todas Formas de Intolerancia y Discriminación fundadas en Religión o Creencia de 1981, que da a las minorías religiosas una protección similar a la que se extendía a grupos raciales, y que en su artículo 6 enuncia derechos que solo pueden ejercerse por un grupo: reunirse en conexión con una religión, enseñar una creencia, difundir publicaciones en estas áreas, etc.

En general, los instrumentos que se han elaborado en el ámbito universal para la protección de las minorías nacionales permiten afirmar que esta se basa en dos 
derechos esenciales: el derecho a la existencia -que implica la obligación de los Estados de no realizar y prohibir toda práctica genocida- y el derecho a la identidad -el genocidio cultural no está penado, pero a la cultura de un grupo la protege el artículo 27 del PIDCP-. Ambos deben interpretarse a la luz del principio de no discriminación, garantizado en varios tratados, parte específica de la ley de las Naciones Unidas y del derecho internacional consuetudinario, pues muchos Estados lo tienen como derecho fundamental.

Según Ruiz Vieytez (2004), los derechos de minorías pueden clasificarse en tres categorías (pp. 516-517). En primer lugar están los que derivan directamente de otros derechos humanos, son individuales y solo exigen abstención de intervención del Estado. Dentro de ellos pueden enumerarse los siguientes derechos: a) desarrollar su identidad religiosa, étnica, lingüística o cultural, b) usar su propia lengua en público y en privado, c) usar un nombre en lengua propia, d) mantener establecimientos educativos específicos, e) establecer contactos con ciudadanos o colectivos de otros países $y, f$ ) no ser discriminado por pertenecer a una comunidad minoritaria.

Por otra parte, las minorías nacionales también tienen derechos que exigen cierta actuación del Estado a través del reconocimiento oficial. Estos son individuales, pero pueden ejercerse en comunidad: usar su idioma en relaciones con instituciones públicas, obtener de las instituciones públicas el uso habitual de su idioma en todo tipo de comunicaciones, recibir instrucción de y en su lengua en instituciones públicas, y obtener apoyo para actividades de promoción de su cultura.

Finalmente, el autor identifica el derecho colectivo a un grado de autogobierno político o administrativo, lo cual se da de acuerdo a ciertas circunstancias e implica el reconocimiento de la personalidad jurídica del grupo titular.

Sobre el contenido de los derechos de las minorías nacionales protegidos en la comunidad internacional contemporánea, Rousso-Lenoir (1996), Lerner (1993) y Klein (1995) plantean distintos listados, que al analizarse arrojan cinco derechos en común: a la existencia, a la identidad cultural, a comunicarse nacional e internacionalmente, a establecer y mantener asociaciones e instituciones, y a la representación política. 
Los cuatro primeros derivan directamente de los derechos humanos y aparecen en los instrumentos fundamentales del derecho internacional de los derechos humanos. De ellos, solo uno tiene relación directa con las minorías: el derecho a proteger y desarrollar la identidad propia. Los otros se garantizan a todos los individuos, y aunque el derecho a la identidad cultural puede relacionarse más específicamente con las minorías, debe definirse de modo más concreto, pues si se reduce a la libertad de pensamiento, consciencia y religión, junto con el derecho a la libre expresión, no añade más a los derechos que el PIDCP garantiza a todas las personas.

La representación política y el reconocimiento jurídico estarían en la tercera categoría de los derechos de minorías nacionales de Ruiz Vieytez, donde chocan con la falta de voluntad de los Estados, aunque son justamente derechos más específicos y de mayor interés para estos colectivos.

En síntesis, puede decirse que los derechos de minorías nacionales reconocidos a nivel universal "son explicitaciones de derechos que se reconocen expresamente a toda persona (p. e. la libertad religiosa) o que disfruta de facto la población mayoritaria (p. e. el derecho a disfrutar la propia cultura)" (Deop Madinabeitia, 2000, p. 435).

En ámbitos regionales, el menor número de Estados, la similitud cultural y las mayores posibilidades de cooperación interestatal por proximidad física, favorecen los acuerdos. Por sus asociaciones históricas con "la cuestión de las minorías", Europa es el mejor punto de partida para un instrumento regional al respecto (Thornberry, 1991, p. 397). Aquí la protección de minorías nacionales se enmarca en el Sistema Europeo de Derechos Humanos, conformado por diversos instrumentos interdependientes (Jimena Quesada, 2006): la Unión Europea, el Consejo de Europa y la Organización para la Seguridad y Cooperación en Europa. Estas coinciden con las tres esferas "geojurídicas" que plantea Toniatti (2000): el derecho supranacional, el derecho internacional y el soft law, que han producido creciente intercambio entre el derecho internacional y los derechos nacionales. Martín-Retortillo (2006) habla de 3 vías europeas de protección de los DD. HH.: la Constitución de cada Estado, el CEDH y los instrumentos UE. Cada uno tiene sus propias reglas, pero al interpretarlos debe considerarse que están interconectados, pues son vías concurrentes: se ven "sometidos 
a intensos flujos recíprocos, con mutuas interferencias que a veces se reflejan en la legislación, acaso en la propia formulación constitucional, y, sobre todo, y más frecuentemente, en la práctica judicial" (p. 55).

La OSCE elaboró los primeros documentos que mencionaron a las minorías nacionales, pero siempre desde el enfoque de la seguridad; pretendía solucionar o evitar conflictos, por lo que tenía una orientación pragmática. Así, sus instrumentos son más políticos que jurídicos, sin que de ellos deriven obligaciones legales sino morales. No son jurídicamente vinculantes, pero algunos creen que en términos prácticos pueden tener incluso mayor impacto o eficacia que otros (Arp. 2008, p. 411). Dentro de ellos puede mencionarse el Acta Final de Helsinki de 1975, cuyo principio 7 incluye la libertad de pensamiento, consciencia, religión o creencia, ejercida "individualmente o en comunidad con otros"; además, hace una valoración positiva del aporte cultural de las minorías nacionales a los Estados. El Documento Final de Copenhague de la Conferencia sobre la Dimensión Humana de 1990, en su artículo 34 ordena a los Estados proteger la identidad cultural de sus minorías nacionales y crear las condiciones para su promoción, y el 35 reconoce su derecho a la participación efectiva en los asuntos públicos. Finalmente, condena el totalitarismo, el odio racial y étnico, el antisemitismo, la xenofobia, la discriminación y la persecución religiosa e ideológica. Además, en las Decisiones de Helsinki de 1992 la OSCE creó el Alto Comisionado para las Minorías Nacionales, instrumento de prevención de conflictos de minorías nacionales en la fase más temprana posible; tiene una función de diplomacia preventiva, no de reparación, y previene conflictos entre Estados partes, no problemas internos de un Estado con alguna minoría en su territorio. Es sobre todo un instrumento de seguridad más que otro vehículo a través del cual puedan dirigirse violaciones de derechos humanos.

El mayor avance que en materia de derechos humanos se ha hecho en Europa y en el mundo, se dio en el Consejo de Europa, con la firma de Convenio Europeo de Derechos Humanos en 1959. Lamentablemente, no tiene ninguna disposición sobre minorías nacionales, pero estas han utilizado su artículo 14 sobre no discriminación, para hacer valer sus derechos. De todos modos, es una cláusula auxiliar más que un derecho independiente, pues una demanda por trato discriminatorio debe invocarse junto con otra por alguna de las disposiciones sustantivas del convenio. 
La importancia del CEDH radica en su naturaleza judicial, lo que evita que su control sea político, pues se centra en la garantía judicial de los derechos humanos a través de sentencias del Tribunal Europeo de Derechos Humanos. Además, es el único mecanismo internacional de derechos humanos donde el individuo puede acceder personalmente a sus órganos, pues tiene locus standi para intervenir directamente ante el tribunal en todas las fases del procedimiento. La labor del TEDH ha sido importantísima para la garantía efectiva y la extensión de los derechos humanos, con una interpretación evolutiva del CEDH en pos de la protección efectiva de derechos concretos, no abstractos. El tribunal ha sido poco receptivo a demandas acerca de modos de vida culturalmente distintos, pero ha estado más dispuesto frente a violaciones a la libertad de expresión, asociación y participación política de minorías. De todos modos, está cada vez más abierto a las demandas de individuos marginados y minorías, aunque los derechos de minorías no estén en el CEDH.

El Consejo de Europa también firmó la Carta Europea para las Lenguas Regionales y Minoritarias en 1992, dedicada a la promoción de las lenguas minoritarias y la diversidad lingüística. No garantiza los derechos generales de miembros de las minorías que hablan estos idiomas, por lo que las protege solo de forma indirecta; tampoco se formulan principios generales sino medidas en ámbitos específicos del uso de las lenguas (educación, administración de justicia, administraciones públicas, vida social y cultural, relaciones transfronterizas y medios de comunicación social). Su sistema de protección es muy flexible para los Estados por la variedad de situaciones en que pueden estar los idiomas no oficiales. El mecanismo de control es a través de informes de los Estados y visitas a los países; las recomendaciones del Comité de Ministros en base a ellos no son jurídicamente vinculantes y no hay sistema sancionatorio. Por ende, es un modelo débil de protección de derechos, pero alumbra derechos exigibles por las minorías.

En 1995, el Consejo de Europa adoptó el Convenio Marco para la Protección de Minorías Nacionales, único instrumento multilateral jurídicamente vinculante dedicado a la protección de estos colectivos. Con esto, se traslada la perspectiva del asunto minorías desde la seguridad hacia la justicia (Craig, 2012). El convenio reconoce derechos individuales, pero algunas disposiciones pueden aplicarse a grupos. Su mecanismo de control se basa en informes periódicos de los Estados al Comité 
de Ministros, asistido por un Comité Consultivo de expertos. Así, los Estados se controlan a sí mismos, lo que cuestiona su efectividad. El órgano encargado de su ejecución -el Comité de Ministros- es un cuerpo político, no judicial. Por eso sus recomendaciones no son jurídicamente vinculantes y así tampoco hay sistema sancionatorio. Por lo tanto, el CMPMN crea obligaciones vinculantes para los Estados, pero no establece un mecanismo supranacional de aplicación, y algunos creen que en esto radica su debilidad (Bautista Jiménez, 1995). Otros justifican esta ausencia de control judicial por su carácter programático, y creen que el CMPMN establece precisamente lo que la situación de las minorías de Europa requiere, por ser tan variada: una doctrina que se sitúe por encima de los Estados, pero considerando que las soluciones a cada caso deben ser diseñadas a medida de cada uno (Weller, 2005, p. 633).

Los Estados mantienen un margen de apreciación amplio, se les obliga en cuanto al resultado, pero pueden adoptar las medidas que consideren oportunas. El CMPMN establece principios y obligaciones generales a los Estados, más que reglas detalladas y derechos que puedan ser invocados por personas pertenecientes a minorías nacionales. De hecho, tiene formulaciones ambiguas y cláusulas escapatorias y faltan definiciones que quedan a criterio de los Estados, incluso la de minoría nacional. De todos modos menciona sus características, especialmente la identidad étnica, cultural, lingüística o religiosa; además, el Comité Consultivo tiene una visión realista, que ha implicado un análisis caso por caso, que ha incorporado a comunidades culturales e históricas.

Para algunos, el CMPMN es un “débil primer intento” (Gilbert, 1996, p. 189), pues la protección que ofrece a las minorías nacionales deriva de la convicción política o de necesidades de estabilidad interna, más que de las exigencias jurídicas del convenio (Ruiz Vieytez, 2004). Para otros, que sea un convenio marco solo implica que no da soluciones únicas a los problemas de las minorías, pues reconoce la amplia variedad de casos. Su enfoque programático puede no ser debilidad sino el camino más lógico (Steketee, 2001), y en ese sentido su importancia legal está entre el hard y el soft law (Craig, 2012). Además, su carácter programático y el amplio margen de apreciación de los Estados explican su éxito por el gran número de estos que lo firmaron (Gál, 2000). 
La Unión Europea, por su parte, tiene una legislación al respecto que se caracteriza principalmente por instrumentos hard law enfocados más en la no discriminación que en la promoción de sus derechos y de medidas de acción positiva. El Tratado de Lisboa de 2007 establece la protección de minorías como vinculante dentro de la Unión Europea, señalando que esta "se fundamenta en los valores de respeto de la dignidad humana, libertad, democracia, igualdad, Estado de derecho y respeto de los derechos humanos, incluidos los derechos de las personas pertenecientes a minorías" (art. 1 bis). Como se observa, tampoco define ni califica a las minorías, ni supera el enfoque individual de los derechos humanos.

La Carta de Derechos Fundamentales del 2000, por otro lado, prohíbe la discriminación por ser miembro de una minoría nacional en su artículo 21, la única disposición que se refiere a ellas. En este considera también razones de raza, color, lengua, religión y origen étnico o social, lo que es muy relevante para las minorías nacionales no reconocidas como tales en sus Estados, que podrían basar en ellas sus demandas. Algo bastante novedoso de la carta es que su artículo 22 establece el respeto de la Unión Europea por la diversidad cultural, religiosa y lingüística como principio de la entidad.

Dentro de la Unión Europea, la Racial Equality Directive del 2000, basada en el principio de trato igualitario sin importar origen étnico o racial, exige a todos los Estados miembros prohibir la discriminación por origen étnico o racial en salud, educación, empleo, protección social, vivienda y acceso a bienes y servicios. Además, en su artículo 2 define por primera vez discriminación directa ("cuando, por motivos de origen racial o étnico, una persona sea tratada de manera menos favorable de lo que sea, haya sido o vaya a ser tratada otra en situación comparable"), discriminación indirecta ("cuando una disposición, criterio o práctica aparentemente neutros sitúe a personas de un origen racial o étnico concreto en desventaja particular con respecto a otras personas, salvo que dicha disposición, criterio o práctica pueda justificarse objetivamente con una finalidad legítima y salvo que los medios para la consecución de esta finalidad sean adecuados y necesarios") y acoso ("constituirá discriminación a efectos de lo dispuesto en el apartado 1 cuando se produzca un comportamiento no deseado relacionado con el origen racial o étnico que tenga como objetivo o consecuencia atentar contra la dignidad de la persona y crear un entorno intimidatorio, 
hostil, degradante, humillante, u ofensivo"). También prohíbe la incitación a la discriminación por origen racial o étnico. En general, establece requerimientos mínimos contra la discriminación, pues el artículo 5 permite a los Estados miembros introducir o mantener medidas de acción positiva, pero no les obliga a ello.

A pesar de todos estos desarrollos, varios de los derechos positivos que se afirman en el Sistema Europeo de Derechos Humanos son versiones del derecho a disfrutar de la cultura propia (Kymlicka, 2007, p. 20). No consideran las características y aspiraciones que distinguen a las minorías nacionales: su consciencia nacional, demandas de autogobierno o la oficialidad de un idioma. El problema es que las minorías nacionales no solo persiguen el derecho individual de sus miembros a practicar su cultura, sino muchas veces también su derecho a autogobernarse.

\section{Protección internacional de los pueblos indígenas}

El derecho internacional sobre pueblos indígenas es nuevo y también se enmarca dentro de la protección internacional de los derechos humanos con instrumentos dedicados a los pueblos originarios, pero también normas relacionadas con derechos de minorías, no discriminación, integridad cultural y propiedad.

Los primeros documentos universales de derechos humanos no incluyen referencias a las comunidades nativas, pero algunas disposiciones han servido de base para el desarrollo posterior de sus derechos. En general, estas pueden usar los mismos instrumentos que sirven a las minorías nacionales para proteger sus derechos, pero siempre y cuando estas sean consideradas como minorías nacionales, étnicas, religiosas o lingüísticas.

El único instrumento dedicado especialmente a estos colectivos, que tiene aplicación universal, es la Declaración de las Naciones Unidas sobre los Derechos de los Pueblos Indígenas, aprobada de forma unánime en el 2007. Aunque solo establece estándares mínimos para su supervivencia, bienestar y dignidad, la participación que tuvieron los indígenas en sus debates y negociaciones le ha dado respaldo de los propios titulares de los derechos que reconoce. La declaración es importantísima 
como primer paso para un avance esencial en la protección de los derechos indígenas, reconoce de manera universal la identidad y entidad de los pueblos originarios, sus derechos particulares y las graves violaciones de derechos humanos que sufren por su condición de tales. Así, prepara el camino para una protección eficaz de sus derechos, pues los codifica en un instrumento ampliamente aceptado que da el marco para su aplicación (Borraz, 2013, pp. 308-309).

Además, tiene un enfoque colectivo -muchas disposiciones establecen a los pueblos indígenas como titulares de ciertos derechos ${ }^{4}-\mathrm{y}$ muestra una conceptualización avanzada de los territorios indígenas no solo como la tierra sino como el hábitat en el cual se desarrolla su cultura.

Por otro lado, en sus artículos 3 y 4 reconoce su derecho a la libre determinación, un avance notable considerando la reticencia de los Estados a reconocerlo. Este ya estaba garantizado en el primer artículo del PIDCP, y en su origen se aplicaba únicamente a los pueblos coloniales en pos de su independencia. Sin embargo, su interpretación ha evolucionado y, como el derecho internacional no define lo que entiende por "pueblos", es difícil delimitar sus titulares. En este sentido, cabe preguntarse si puede aplicársele a los pueblos originarios.

Sin embargo, las comunidades autóctonas no demandan independencia. Ciertamente han utilizado su derecho a la autodeterminación para exigir un trato distinto al de las minorías, de las que se distinguen por razones relacionadas con su pasado colonial; las invasiones y despojos que sufrieron son la base para reclamar la restitución de derechos perdidos, no la protección de derechos concedidos, y sus antepasados fueron naciones soberanas, sometidas e incorporadas contra su voluntad a Estados extrańos (Stavenhagen, 1992, p. 95). Pero al reclamar su derecho a la autodeterminación, se han referido solo a la administración de sus tierras ancestrales, su supervivencia como grupos no depende solo de que se les reconozcan sino también de que se les permita administrarlas y tomar decisiones sobre asuntos que les afecten directamente.

4 El artículo 1, por ejemplo, dice que los indígenas tienen derecho, como pueblos o como individuos, al disfrute pleno de todos los derechos humanos; el 26, que los pueblos indígenas tienen derecho a las tierras, territorios y recursos que tradicionalmente han poseído, ocupado o utilizado o adquirido. 
Por otra parte, la declaración contiene la tradicional cláusula de protección de la integridad territorial del Estado en su artículo 46:

\begin{abstract}
Nada de lo contenido en la presente declaración se interpretará en el sentido de que confiere a un Estado, pueblo, grupo o persona derecho alguno a participar en una actividad o realizar un acto contrarios a la Carta de las Naciones Unidas, ni se entenderá en el sentido de que autoriza o alienta acción alguna encaminada a quebrantar o menoscabar, total o parcialmente, la integridad territorial o la unidad política de Estados soberanos e independientes.
\end{abstract}

Además, en su formulación especifica que "tienen derecho a la autonomía o el autogobierno en las cuestiones relacionadas con sus asuntos internos y locales, así como los medios para financiar sus funciones autónomas" (art. 4). Es decir, no reconoce un derecho de secesión, y el artículo 5 lo aclara: "tienen derecho a conservar y reforzar sus propias instituciones políticas, jurídicas, económicas, sociales y culturales, manteniendo a la vez su derecho a participar plenamente, si lo desean, en la vida política, económica, social y cultural del Estado”.

Por todo lo expuesto, el conjunto normativo que protege a los pueblos indígenas en el ámbito universal se basa en normas pertenecientes a cinco categorías (Anaya, 2005, p. 32): no discriminación, integridad cultural, tierras y recursos naturales, bienestar social y desarrollo, y autogobierno, como dimensión política de la autodeterminación, que implica cierta autonomía y participación política efectiva sin violar la integridad territorial del Estado.

Un instrumento muy relevante, desarrollado dentro del marco de las Naciones Unidas pero sin aplicación universal, es el Convenio n. ${ }^{\circ} 169$ sobre Pueblos Indígenas y Tribales en Países Independientes de la Organización Internacional del Trabajo, firmado en 1989 y ratificado por 22 Estados; hoy es el único instrumento jurídico internacional obligatorio dedicado íntegramente a los pueblos indígenas. Uno de sus aportes más significativos es que usa la palabra pueblos en su artículo 1, aunque eso "no deberá interpretarse en el sentido de que tenga implicación alguna en lo que atañe a los derechos que pueda conferirse a dicho término en el derecho internacional”. En 
la misma disposición, incluye la autoidentificación como criterio fundamental para su definición.

El Convenio n. ${ }^{\circ} 169$ reconoce derechos políticos y territoriales de los pueblos indígenas desde una perspectiva individual y colectiva. Dentro de los primeros, destaca su derecho a la participación y consulta (arts. 2 y 6), que requiere establecer procedimientos adecuados para llegar a un acuerdo u obtener su consentimiento, aunque ninguno de ellos sea estrictamente necesario. Según Magdalena Gómez (2006, p. 149), esto significa que los pueblos originarios no tienen la posibilidad de negarse de manera efectiva y con consecuencias, a una actividad que les afecte. Sin embargo, cabe recordar que el procedimiento debe siempre realizarse de buena fe, con miras a ese acuerdo o consentimiento.

Por otro lado, el artículo 7 se refiere a la autogestión y autogobierno, afirmando su derecho a controlar su desarrollo económico, social y cultural -"en la medida de lo posible" - y a participar en los proyectos de desarrollo que les afecten. Relacionado con esto, el artículo 8 garantiza su derecho a conservar sus costumbres e instituciones.

Los derechos territoriales están garantizados en la segunda parte del convenio, en base al respeto por la relación de los pueblos originarios con sus territorios y a un concepto amplio de estos que incluye la tierra, las aguas, el espacio aéreo, el medio ambiente, las lagunas sagradas y los centros ceremoniales (art. 13). El artículo 14 del convenio reconoce el derecho de las comunidades indígenas a la propiedad y posesión de las tierras que tradicionalmente ocupan y a utilizar tierras que no estén exclusivamente ocupadas por ellos pero a las que hayan tenido tradicionalmente acceso para sus actividades tradicionales y de subsistencia. Los Gobiernos deben determinar esas tierras y establecer procedimientos adecuados para solucionar reivindicaciones indígenas.

En cuanto a los recursos naturales de sus territorios, los indígenas tienen derecho a participar en su utilización, administración y conservación (art. 15). Cuando los recursos del subsuelo pertenecen al Estado, los Gobiernos deben consultarles antes de realizar un programa de prospección o explotación de ellos. Además, los pueblos 
originarios deben participar de los beneficios de esas actividades - "en lo posible"- y ser indemnizados si estas les causan algún daño. Finalmente, las comunidades nativas no deben ser trasladadas de las tierras que ocupan, y si es necesario, se requiere su consentimiento libre e informado (art. 16).

Entre los artículos 8 y 12 se tratan las relaciones entre el derecho consuetudinario indígena y el sistema jurídico estatal, mencionando el derecho de los pueblos originarios a conservar su orden normativo interno y a que sus costumbres sean consideradas al aplicarles la legislación nacional. Es decir, no se juzgará en base a ellas, pero se tendrán en cuenta.

El mecanismo de control del convenio se basa, en primer lugar, en informes periódicos de los Estados al Comité de Expertos de la OIT. Si un Estado incumple el convenio, pueden aplicarse mecanismos de presión diplomática indirecta. Además, según la Constitución de la OIT (1919), las organizaciones de trabajadores o empleadores pueden presentar reclamos por incumplimiento del convenio por parte de un Estado miembro; también puede un Estado presentar una queja contra otro (arts. 24 y ss.). En estos casos, una comisión estudia el reclamo y redacta un informe; el Estado puede aceptar o no las recomendaciones, y en caso negativo pueden someter la queja a la Corte Internacional de Justicia. Las organizaciones indígenas no pueden presentar una reclamación, pero sus miembros pueden unirse a organizaciones de trabajadores y empleadores y en la práctica han podido presentar informaciones, comentarios y reclamos.

Dentro de las limitaciones del convenio, destaca su lenguaje, pues relativiza los derechos reconocidos con frases como "cuando sea apropiado" o "en lo posible". Además, tiene disposiciones programáticas muy generales y su mecanismo de aplicación se centra en informes estatales y sanciones morales. De todos modos, es esencial en el reconocimiento de los derechos indígenas, especialmente en América Latina, siendo muy utilizado por estos pueblos para plantear sus demandas. De hecho, ha influido en las políticas indigenistas de los países que lo han ratificado -en la región hay una tendencia hacia el "constitucionalismo multicultural", en la cual ha influido bastante el convenio (Van Cott, 2000)-, y ha sido referencia para 
otros instrumentos internacionales, como las Declaraciones sobre los Derechos de los Pueblos Indígenas firmadas en el ámbito universal de la ONU y en el entorno regional de la Organización de Estados Americanos (OEA). Lamentablemente, ha resultado ser el paso máximo que están dispuestos a dar los Estados, y son pocos los que lo han ratificado, algo preocupante siendo el único instrumento jurídico internacional vinculante que trata directamente los derechos indígenas.

En el ámbito interamericano es donde mayor desarrollo ha tenido la protección internacional de los derechos indígenas. Así como sucede en Europa con la de las minorías nacionales, en este caso los derechos de los pueblos originarios en la región americana están protegidos dentro del sistema regional de derechos humanos, en el marco de la labor de la OEA al respecto. Por lo tanto, están garantizados en sus instrumentos básicos. En primer lugar está la Declaración Americana sobre los Derechos y Deberes del Hombre de 1948, que contiene los derechos básicos de todos los instrumentos de derechos humanos. Destaca su artículo 23, que establece que "toda persona tiene derecho a la propiedad privada correspondiente a las necesidades esenciales de una vida decorosa, que contribuya a mantener la dignidad de la persona y el hogar". Esta explicación, que no aparece en la DUDH, puede servir a las reivindicaciones territoriales indígenas, aunque está solo en su dimensión individual.

Los derechos indígenas también están garantizados en la Convención Americana sobre los Derechos Humanos de 1969, principal instrumento de protección de derechos humanos en el Sistema Interamericano. No menciona los derechos indígenas ni se dedica a su protección, pero establece un mecanismo de control que incluye, además de la Comisión Interamericana de Derechos Humanos (CIDH), un tribunal internacional específico para conocer las violaciones de sus disposiciones: la Corte Interamericana de Derechos Humanos (CorteIDH).

La CIDH tiene competencias genéricas para proteger los derechos humanos y puede hacer recomendaciones que tienen autoridad e influencia, pero que no son jurídicamente vinculantes. Ha tenido un rol más relevante en su función contenciosa, pues es la primera instancia del proceso de peticiones individuales que debe ser agotada 
antes de que la CorteIDH las conozca. Esta última tiene competencias consultivas y contenciosas. Hasta ahora no se ha requerido su pronunciamiento acerca de asuntos indígenas, pero en su función contenciosa se le han presentado casos de violación de derechos indígenas, cuyas sentencias han contribuido enormemente al reconocimiento y la configuración de esos derechos. Su jurisprudencia al respecto comenzó con el caso entre la comunidad mayagna (sumo) awas tingni $v$ s. Nicaragua, cuyo fallo fue "el primer pronunciamiento tan categórico de un órgano internacional de DD. HH.” sobre los derechos colectivos de las comunidades nativas (Rodríguez-Piñero, 2008, pp. 114-115). El caso awas tingni se origina en un proyecto de extracción forestal en 1992, por el cual la comunidad de 600 personas firmó un acuerdo con la empresa Madensa para reconocer ciertos derechos de participación de los indígenas sobre el territorio por su ocupación ancestral, y establecer el manejo integral del bosque. Más tarde, por medio de un convenio entre ambas partes y el Ministerio de Ambiente y Recursos Naturales de Nicaragua, este último se comprometió a delimitar las tierras comunales. Sin embargo, en 1996 el Estado dio una concesión a la empresa Solcarsa para explotar por 30 años 62 mil hectáreas de la zona, sin consultar previamente a la comunidad awas tingni. Tras infructuosas peticiones de no continuar con la concesión y de definir los territorios, esta presentó el caso ante la CIDH, que dictó sentencia de fondo en agosto de 2001.

La disputa incluyó algunos de los aspectos más relevantes de los derechos indígenas: su relación con la tierra (derecho de propiedad colectiva, delimitación de tierras, derecho a sus recursos) y su derecho a participar en asuntos que les afecten directamente (derecho a consulta). Desde entonces, la CorteIDH ha fallado en más de veinte casos de derechos indígenas, desarrollando una jurisprudencia completa y precisa al respecto que incluye el reconocimiento de varios derechos fundamentales para los pueblos originarios, dándoles interpretaciones que incluso los amplían.

En primer lugar, el tribunal ha reconocido el derecho a la vida que la CADH garantiza en su artículo 4, interpretándolo con miras a la obligación del Estado de asegurar el medio ambiente natural a los pueblos indígenas por su relación especial con la tierra y la fuerte dependencia de sus recursos naturales; su pérdida, por lo tanto, amenaza la preservación de sus costumbres y su existencia física. Así, ha enfatizado los aspectos 
materiales y culturales del derecho a la vida, señalando que este peligra por los efectos de la explotación industrial de las tierras (Anaya, 2006, p. 47).

Por otro lado, la Corte ha reconocido el derecho de los pueblos originarios a la propiedad de la tierra y sus recursos. El derecho a la propiedad está garantizado en el artículo 21 de la CADH desde una perspectiva individual. En cambio, la CorteIDH ha desarrollado un enfoque multidimensional para proteger los derechos territoriales indígenas, tanto en una dimensión colectiva del derecho de los pueblos indígenas a la integridad cultural, como en la dimensión individual de los derechos de sus miembros al bienestar físico, mental y social (Tramontana, 2010, p. 249). Esta doctrina es una de sus contribuciones más significativas (Campbell, 2013, p. 117). Además, como se mencionó, vincula el derecho a la propiedad colectiva de los pueblos originarios con su derecho a la vida y ha desarrollado un concepto amplio de ella, similar a la idea de territorio del Convenio n. ${ }^{\circ} 169$ de la OIT.

Por otrolado, el tribunal ha destacado el deber de considerar el derecho consuetudinario de los pueblos indígenas, por lo que no debería ser necesario un título sobre la propiedad para reconocer y registrar oficialmente una tierra que posean de modo efectivo. Con esto, reconoce implícitamente cierto derecho de autodeterminación de las comunidades indígenas y establece su derecho a regirse por sus propios regímenes normativos.

Las reclamaciones de tierras indígenas pueden presentar un elemento bastante complejo, el choque entre propiedad particular y colectiva. En muchos casos los predios reclamados están en manos de terceros inocentes que los han comprado y también tienen legítimo derecho sobre ellos. Al respecto, la CorteIDH afirma que los pueblos aún conservan derecho a reclamarlos, pues los habían perdido por motivos ajenos a su voluntad (caso yakye axa vs. Paraguay, 2006). La explotación productiva de las tierras por sus propietarios actuales no anula este derecho, pues esa perspectiva aborda la cuestión "exclusivamente a través de la productividad de la tierra y del régimen agrario, lo que resulta ineficiente a las peculiaridades propias de dichos pueblos" (caso sawhoyamaxa vs. Paraguay, 2006, párr. 139). La relación especial de los pueblos con sus tierras es fundamento para determinar hasta cuándo tienen 
derecho para reclamarlas: "mientras esa relación exista, el derecho a la reivindicación permanecerá vigente, caso contrario, se extinguirá”. Ese vínculo además es amplio y contempla un uso productivo pero también cultural y espiritual, tanto de forma permanente como esporádica (caso sawhoyamaxa vs. Paraguay, 2006, párr. 131).

Sobre la contradicción entre la propiedad privada de los nuevos propietarios y la colectiva de la comunidad indígena, la Corte recuerda las restricciones permitidas para su ejercicio: deben estar establecidas por ley, ser necesarias, proporcionales, y hacerse para un objetivo legítimo en una sociedad democrática. Entonces, la ley puede subordinar el uso y goce de los bienes al interés social ya que los objetivos colectivos priman sobre el disfrute pleno del derecho restringido. En consecuencia, el Estado debe valorar en cada caso las eventuales restricciones del reconocimiento de uno sobre otro. Así, debe considerar que la propiedad colectiva es para los indígenas un concepto más amplio que el de la propiedad privada individual, pues se relaciona con la conservación de su patrimonio cultural y supervivencia. Por lo tanto, desconocerles ese derecho podría afectar otros, como el derecho a la identidad cultural o incluso a la vida. En cambio, restringir el derecho a la propiedad privada de particulares puede ser necesario para el objetivo colectivo de preservar las identidades culturales en una sociedad democrática y pluralista, y proporcional, si se paga una justa indemnización. Si por motivos razonables el Estado no puede devolver los territorios a los indígenas, debe entregarles tierras alternativas de igual tamaño y calidad -elegidas con su consentimiento y según sus medios de consulta-, una indemnización justa o ambos (caso xákmok kásek vs. Paraguay, 2010).

En síntesis, los planteamientos de la CorteIDH sobre el derecho de propiedad indígena son los siguientes: i) la posesión tradicional de los indígenas sobre sus tierras tiene efectos equivalentes al título de pleno dominio que otorga el Estado, ii) su posesión tradicional también les da derecho a exigir el reconocimiento oficial de propiedad y su registro 5 , iii) los indígenas que han perdido involuntariamente la posesión de sus tierras tradicionales mantienen el derecho de propiedad sobre ellas, aun sin título legal, salvo que hayan sido legítimamente trasladadas a terceros y, iv)

5 La identificación, demarcación y entrega de títulos de propiedad es esencial para garantizar los derechos indígenas sobre sus territorios, pues la incertidumbre que resulta de su ausencia facilita su violación por los Estados. 
si esas tierras se han trasladado legítimamente a terceros inocentes, tienen derecho a recuperarlas u obtener otras de igual extensión y calidad ya que la posesión no condiciona su derecho a la recuperación.

Una forma de vulnerar los derechos indígenas más sutil que el despojo de sus territorios o su eliminación como grupo, es no respetar sus derechos políticos. Estos los garantiza el artículo 23.1 de la $\mathrm{CADH}$ para todos los ciudadanos, pero a los pueblos originarios se les ha limitado en casi toda la región, lo que repercute en el ejercicio de su derecho de autodeterminación. La CorteIDH se pronunció al respecto en el caso Yatama contra Nicaragua (2005), enfocándose en el carácter especial de la comunidad indígena a la que Yatama representaba y sus necesidades particulares como minoría étnica. Al respecto, señaló que la ley electoral del país disminuyó las posibilidades de participación de las organizaciones indígenas (por ejemplo, solo permitía que participaran partidos políticos, forma de organización que no es propia de los pueblos indígenas). El enfoque de la CorteIDH aquí también es amplio, considera las particularidades de las comunidades indígenas, más que las formalidades, pues el foco está en el goce efectivo de los derechos. Además, responde a una visión positiva de los derechos humanos que obliga al Estado a adoptar medidas para dar igualdad de oportunidades a personas y grupos: mecanismos que incluyan cuotas étnicas, órganos consultivos, regiones autónomas, circunscripciones especiales, etc. Además, relacionó la violación del derecho a ser elegido con el derecho a votar. Los electores no pudieron votar por los candidatos excluidos, limitando su derecho al sufragio.

Un derecho político reconocido especialmente para los pueblos originarios, es su derecho a la consulta previa. Este deriva de los otros derechos mencionados: por un lado, es una forma de aplicar su derecho a la participación; por otro, deriva de sus derechos territoriales. Si estos son restringidos por un proyecto industrial, el Estado debe garantizar su participación en todas las etapas de su desarrollo dentro de su territorio, consultándoles según sus costumbres y métodos de toma de decisiones, en las primeras etapas del proyecto, de buena fe y con el fin de lograr un acuerdo. Según la Corte, en proyectos de gran escala con mayor impacto en la comunidad, el Estado debe también obtener el consentimiento previo, libre e informado, de acuerdo con sus tradiciones, aspecto en el cual va más allá del Convenio n. ${ }^{\circ} 169$ de la OIT y de 
la Declaración de las Naciones Unidas. Además, debe realizar estudios previos de impacto ambiental y social y garantizar que el pueblo obtenga un beneficio razonable de esa actividad. Este derecho está en el Convenio n. ${ }^{\circ} 169$ de la OIT y en la ley interna de muchos países americanos, y la CorteIDH afirmó que "es también un principio general del derecho internacional” (caso sarayaku vs. Ecuador, 2012, párr. 164). Pero su puesta en práctica depende de la voluntad de los Estados, y muchos ven la consulta solo como mecanismos formales, y si la repuesta es negativa respecto al proyecto, no se considera.

Berraondo (2013) ha sintetizado la doctrina de la CorteIDH sobre derechos indígenas interrelacionados, diciendo que

[...] los derechos territoriales mantienen una estrecha relación con los derechos culturales y espirituales, con el derecho a la vida digna, a la integridad física de las personas, a la libertad de circulación y residencia, a la autodeterminación, a la participación efectiva, y con los derechos de consulta y consentimiento previo, libre e informado. [...] Asociados a los derechos territoriales nos encontramos con un derecho de propiedad, un derecho de titulación y demarcación y un derecho de restitución, compensación e indemnización (p. 197).

De esa forma, el tribunal ha interpretado los derechos reconocidos en la $\mathrm{CADH}$ de manera favorable a los pueblos originarios, aunque el tratado no contiene disposiciones relativas a ellos.

El primer instrumento americano dedicado a los derechos indígenas es la Declaración Americana sobre los Derechos de los Pueblos Indígenas, aprobada en el 2016. Esta no contiene una definición de "pueblos indígenas", pero da gran importancia a su autoidentificación en su artículo 1 . El artículo 2 se refiere a la pluriculturalidad, pero solo reconoce el carácter pluricultural y multilingüe de los pueblos originarios, mas no de los Estados, cuya diversidad cultural se debe justamente a la presencia de estos grupos dentro de sus fronteras (IWGIA, 2016). 
La segunda sección se refiere a los "derechos humanos y derechos colectivos", reconociendo al grupo como titular de derechos; estos se enuncian en el artículo 6, pero sin mayor desarrollo. Llama la atención que, aunque se reconocen derechos de autonomía -a sus sistemas jurídicos, sociales, políticos y económicos-, no se menciona el derecho de autodeterminación en sí. Este aparece en el artículo 3, en los mismos términos de la Declaración de las Naciones Unidas de 1992. Separado de esto, el artículo 21.1 afirma su "derecho a la autonomía o al autogobierno en las cuestiones relacionadas con sus asuntos internos y locales, así como a disponer de medios para financiar sus funciones autónomas". Es decir, la declaración americana separa las disposiciones sobre autodeterminación y autonomía, y los excluye de los derechos colectivos del artículo 6, donde solo afirma que "los Estados promoverán con la participación plena y efectiva de los pueblos indígenas la coexistencia armónica de los derechos y sistemas de los grupos poblacionales y culturas".

Los derechos territoriales de los pueblos indígenas están reconocidos en el artículo 25 de la declaración americana: "tienen derecho a las tierras, territorios y recursos que tradicionalmente han poseído, ocupado o utilizado o adquirido". Así, omite el derecho de propiedad ancestral de acuerdo con una ocupación histórica pero no actual de la tierra. La CorteIDH se ha manifestado a favor de este derecho, por lo que la declaración daría un paso atrás en este aspecto. La disposición también se refiere a la delimitación y los títulos de las tierras:

Los pueblos indígenas tienen el derecho al reconocimiento legal de las modalidades y formas diversas y particulares de propiedad, posesión o dominio de sus tierras, territorios y recursos de acuerdo con el ordenamiento jurídico de cada Estado y los instrumentos internacionales pertinentes. Los Estados establecerán los regímenes especiales apropiados para este reconocimiento y su efectiva demarcación o titulación.

Por esta razón, el que decide el modo de demarcar y reconocer jurídicamente los derechos territoriales de los pueblos indígenas es el Estado (respetando sus costumbres y sistemas de tenencia de la tierra), pero no ellos mismos en ejercicio de su libre determinación. 
La participación y el derecho a la consulta previa están en la declaración americana, pero no en relación con la propiedad de la tierra y los recursos. No se menciona al tratar los derechos territoriales de los pueblos indígenas, sino, primero, su derecho a la participación (art. 23: "los Estados celebrarán consultas y cooperarán de buena fe con los pueblos indígenas interesados por medio de sus instituciones representativas antes de adoptar y aplicar medidas legislativas o administrativas que los afecten, a fin de obtener su consentimiento libre, previo e informado"), a la protección de su patrimonio cultural y propiedad intelectual (art. 28) -para la protección cultural y de la propiedad intelectual de los indígenas, señala que los Estados realizarán consultas encaminadas a obtener su consentimiento-, y al derecho al desarrollo (art. 29). El derecho al desarrollo está redactado de modo tal, que se advierte su relación con la autodeterminación, aunque no se nombra: "Los pueblos indígenas tienen derecho a mantener y determinar sus propias prioridades en lo relacionado con su desarrollo político, económico, social y cultural, de conformidad con su propia cosmovisión. Asimismo, tienen el derecho a que se les garantice el disfrute de sus propios medios de subsistencia y desarrollo y a dedicarse libremente a todas sus actividades económicas" (art. 29.1). De todos modos, no establece la obligatoriedad de su consentimiento, pero sí de realizar las consultas con miras al acuerdo con las comunidades.

Luego, la declaración americana se refiere a su derecho a "participar activamente en la elaboración y determinación de los programas de desarrollo que les conciernan y, en lo posible, administrar esos programas mediante sus propias instituciones" (art. 29.3). De eso deriva su derecho a ser consultadas previamente y la obligación de los Estados de realizar estos procedimientos (art. 29.4), lo que no se vincula con sus derechos territoriales.

Así, en algunos aspectos la declaración americana no avanzó como se esperaba, pero reconoce reclamaciones significativas para los pueblos originarios. Representa un consenso muy importante y difícil de alcanzar y muestra la opinio iuris americana al respecto. Además, surgiendo en el ámbito de la OEA, tiene un marco de presión moral y política para su cumplimiento. No obstante, es solo una declaración no vinculante y sin mecanismos eficaces de control. Se adoptó hace muy poco tiempo, por lo que sus efectos aún están por verse. En las últimas décadas ha habido un gran avance en el plano normativo; los esfuerzos deberían dedicarse ahora a su aplicación 
para que sean efectivos. Ante esto hay varios obstáculos por la falta de voluntad de los Estados.

\section{Conclusiones}

Minorías nacionales y pueblos indígenas son grupos étnicos con muchos elementos en común, pero también con ciertas diferencias relevantes. Esto ha llevado a que la protección internacional de sus derechos haya tomado caminos distintos. En el sistema universal, ambos pueden beneficiarse de los mismos instrumentos de derechos humanos en general. Sin embargo, la Asamblea General de las Naciones Unidas ha elaborado declaraciones para cada uno por separado. En el ámbito regional, las minorías nacionales han usado los instrumentos desarrollados dentro del Sistema Europeo de Derechos Humanos, y los pueblos indígenas, los del Sistema Interamericano de Derechos Humanos. En ambos casos, cuentan con instrumentos de derechos humanos en general y de otros dedicados a ellos de modo específico, que enfatizan en la protección de su identidad cultural y establecen obligaciones positivas para los Estados al respecto.

En cuanto al contenido de los derechos protegidos, puede decirse que "los pueblos indígenas tienen todos los derechos que tienen las minorías, pero además pueden tener el derecho a la autodeterminación con todos sus problemas de interpretación y aplicación" (Castellino, 2010, p. 396) ${ }^{6}$. Si bien algunas minorías nacionales han ganado ciertos derechos de autonomía y autogobierno, el de la libre determinación es propio de las comunidades nativas por el hecho de ser "pueblos", y lo garantiza el derecho internacional a todos los pueblos indígenas, no solo a algunos. Además, de ese derecho han surgido otros que son propios de ellos por sus características esenciales, tales como los derechos territoriales, el derecho a la consulta previa y el rol de la autoidentificación en su definición como pueblos indígenas.

6 "Indigenous peoples have all the rights that minorities have, but may in addition also have the right to selfdetermination with all its attendant problems of interpretation and application”. Traducción propia. 
En cuanto a la aplicación de esos derechos, hay falencias significativas en la efectividad del sistema actual para ambos grupos, pues la erosión de la soberanía estatal que hay en el derecho internacional en general, no se ha extendido de la misma manera a la protección de pueblos indígenas y minorías nacionales.

\section{REFERENCIAS}

Aguilar, G. (2007). La dinámica internacional de la cuestión indigena. Santiago de Chile, Chile: Librotecnia.

AGNU. (1948). Resolución 260 A (III), Convención sobre la Prevención y Castigo del Crimen de Genocidio. Adoptada el 9 de diciembre de 1948.

Anaya, J. (2005). Los pueblos indigenas en el derecho internacional. Madrid, España: Trotta.

Anaya, J. (2006). Los derechos de los pueblos indígenas. En M. Berraondo (coord.), Pueblos indigenas y derechos humanos (pp. 29-60). Bilbao, España: Universidad de Deusto.

Arp, B. (2008). Las minorias nacionales y su protección en Europa. Madrid, España: Centro de Estudios Políticos y Constitucionales.

Bautista Jiménez, J. M. (1995). El Convenio Marco para la Protección de las Minorías Nacionales: construyendo un sistema europeo de protección de las minorías. Revista de Instituciones Europeas, 22(3), 939-960.

Berraondo, M. (2013). Derechos territoriales frente al reto permanente de su implementación. En F. Gómez Isa y M. Berraondo (eds.), Los derechos indígenas tras la Declaración: El desafio de la implementación (pp. 191-205). Bilbao, Espańa: Universidad de Deusto.

Borraz, P. (2013). Después de la Declaración: perspectivas en la participación indígena en las Naciones Unidas. En F. Gómez Isa y M. Berraondo (eds.), Los derechos indígenas tras la Declaración: El desafio de la implementación (pp. 287-321). Bilbao, España: Universidad de Deusto. 
Campbell, M. S. (2013). Contribución del sistema interamericano en la definición e interpretación de la Declaración de la ONU sobre los Derechos de los Pueblos Indígenas. En F. Gómez Isa y M. Berraondo (eds.), Los derechos indígenas tras la Declaración: El desafío de la implementación (pp. 113-135). Bilbao, España: Universidad de Deusto.

Capotorti, F. (1979). Study on the rights of persons belonging to ethnic, religious and linguistic minorities. Nueva York: Naciones Unidas.

Castellino, J. (2010). The protection of minorities and indigenous peoples in international law: a comparative temporal analysis. International Journal on Minority and Group Rights, 17(3), 393-433.

Conferencia Mundial de Derechos Humanos. (1993). Declaración y Programa de Acción de Viena. Adoptada el 25 de junio de 1993.

CorteIDH. (2001). Caso de la comunidad mayagna (sumo) awas tingni vs. Nicaragua. Fondo, reparaciones y costas. Sentencia del 31 de agosto de 2001. Serie C n. 79.

CorteIDH. (2005). Caso comunidad indígena yakye axa vs. Paraguay. Fondo, reparaciones y costas. Sentencia del 17 de junio de 2005. Serie C n. 125.

CorteIDH. (2005). Caso Yatama vs. Nicaragua. Excepciones preliminares, fondo, reparaciones y costas. Sentencia del 23 de junio de 2005. Serie C n. ${ }^{o} 127$.

CorteIDH. (2006). Caso comunidad indígena sawhoyamaxa vs. Paraguay. Fondo, reparaciones y costas. Sentencia del 29 de marzo de 2006. Serie C n. ${ }^{\circ} 146$.

CorteIDH. (2010). Caso comunidad indígena xákmok kásek vs. Paraguay. Fondo, reparaciones y costas. Sentencia del 24 de agosto de 2010. Serie C n. 214.

CorteIDH. (2012). Caso pueblo indígena kichwa de sarayaku vs. Ecuador. Fondo y reparaciones. Sentencia del 27 de junio de 2012. Serie C n. ${ }^{\circ} 245$. 
Craig, E. (2012). From Security to Justice? The Development of a More Justice-oriented Approach to the Realisation of European Minority Rights Standards. Netherlands Quarterly of Human Rights, 30(1), 41-65. Recuperado de http://sro.sussex.ac.uk/38581/1/ NQHR2012FINAL.pdf

Daes, É.-I. (1996) Documento de trabajo sobre el concepto de "pueblos indígenas". Grupo de Trabajo sobre Poblaciones Indígenas, $14^{\circ}$ período de sesiones, E/CN.4/Sub.2/ AC.4/1996/2.

Deop Madinabeitia, X. (2000). La protección de las minorías nacionales en el Consejo de Europa. Bilbao, España: Instituto Vasco de Administración Pública.

Eriksen, T. (2002). Ethnicity and Nationalism. Anthropological Perspectives (2a ed.). Londres, Inglaterra: Pluto Press.

Gál, K. (2000). The Council of Europe Framework Convention for the Protection of National Minorities and its impact on Central and Eastern Europe. Journal on Ethnopolitics and Minority Issues in Europe, 1, 1-17. Recuperado de http://www.ecmi.de/fileadmin/ downloads/publications/JEMIE/JEMIE05Gal30-07-01.pdf

George, P. (1985). Geopolitica de las minorias. Barcelona, España: Oikos-Tau.

Gilbert, G. (1996). The Council of Europe and minority rights. Human Rights Quarterly, 18, 160-189.

Gómez, M. (2006). El Convenio 169 de la Organización Internacional del Trabajo. En M. Berraondo (coord.), Pueblos indígenas y derechos humanos (pp. 133-151). Bilbao, España: Universidad de Deusto.

Gómez del Prado, J. L. (2002). Pueblos indígenas. Normas internacionales y marcos nacionales. Bilbao, España: Universidad de Deusto. 
IWGIA (Grupo Internacional de Trabajo sobre Asuntos Indígenas) y Observatorio Ciudadano de Chile. (2016). Análisis: Declaración Americana de Derechos de los Pueblos Indígenas. Recuperado de http://www.iwgia.org/noticias/buscar-noticias?news_id=1371.

Jimena Quesada, L. (2006). Sistema europeo de derechos fundamentales. Madrid, España: Colex.

Klein, E. (1995). Minderheitenschutz im Völkerrecht. En G. Baadte y A. Rauscher (eds.) Minderheiten, Migration und Menschenrechte (pp. 127-153). Graz, Austria: Styria.

Kymlicka, W. (1996). Ciudadanía multicultural. Barcelona, España: Paidós.

Kymlicka, W. (2007). La evolución de las normas europeas sobre los derechos de las minorías: los derechos a la cultura, la participación y la autonomía. Revista Española de Ciencia Politica, (17), 11-50. Recuperado de https://recyt.fecyt.es/index.php/recp/article/ view/37447/20965

Lemkin, R. (1944). Axis rule in occupied Europe. Washington D. C.: Carnegie Endowment for International Peace.

Lerner, N. (1993). The evolution of minority rights in International law. En C. Brölmann, R. Lefeber y M. Zieck (eds.), Peoples and minorities in international law (pp. 77-101). Dordrecht, Países Bajos: Martinus Nijhoff Publishers.

Martín-Retortillo, L. (2006). Vías concurrentes para la protección de los derechos humanos: Perspectivas española y europeas. Cizur Menor, España: Aranzadi.

Martínez Cobo, J. R. (1987). Study of the Problem of the Discrimination Against Indigenous Populations, Nueva York: Naciones Unidas.

OIT. (1919). Constitución de la OIT. Aprobada el 11 de abril de 1919.

Pentassuglia, G. (2002). Minorities in International Law. Estrasburgo, Francia: Council of Europe Publishing. 
Rodríguez-Piñero, L. (2008). La OIT y los pueblos indígenas: una introducción histórica. En J. E. R. Ordóńez e I. Zaragoza (coords.), Pueblos indígenas y tribales: respeto, participación y consulta (pp. 105-132). México D. F., México: Plaza y Valdés.

Roth, S. (1992). Toward a Minority Convention: its need and content. En Y. Dinstein y M. Tabory (eds.), The protection of minorities and human rights (pp. 83-116). Dordrecht, Países Bajos: Martinus Nijhoff Publishers.

Rousso-Lenoir, F. (1996) ¿Derechos de las minorías o igualdad? En L. Prieto Sanchís (coord.), Tolerancia y minorias: problemas jurídicos y politicos de las minorías en Europa (pp. 135145). Cuenca, Espańa: Ediciones de la Universidad de Castilla-La Mancha.

Ruiz Vieytez, E. (2004) El Convenio-Marco para la Protección de las Minorías Nacionales. En F. Gómez Isa (dir.) y J. M. Pureza, La protección internacional de los derechos humanos en los albores del siglo XXI (pp. 513-526). Bilbao, España: Universidad de Deusto.

Sarivaara, E., Maatta, K., y Uusiautti, S. (2013). Who is indigenous? Definitions of indigeneity. European Scientific Journal, 1 (edición especial), 369-378. Recuperado de https:// eujournal.org/index.php/esj/article/view/2317/2190

Sobero, Y., y R. Calduch (dir.) (2006). Conflictos étnicos: el caso de los pueblos indígenas. Madrid, España: Universidad Complutense de Madrid.

Stavenhagen, R. (1992). Los derechos indígenas. Algunos problemas conceptuales. Revista IIDH, 15, 123-143.

Steketee, F. (2001). The Framework Convention: a piece of art or a tool for action? International Journal on Minority and Group Rights, 8(1), 1-15.

Thornberry, P. (1991). International Law and the rights of minorities. Oxford, Inglaterra: Clarendon Press.

Thornberry, P. (1995). The UN Declaration on the Rights of Persons Belonging to National or Ethnic, Religious and Linguistic Minorities: Background, Analysis, Observations, and 
an Update. En A. Phillips y A. Rosas (eds.), Universal minority rights (pp. 13-76). Turku/ Åbo, Finlandia: Åbo Akademi University Institute for Human Rights.

Toniatti, R. (2000). Los derechos del pluralismo cultural en la nueva Europa. Revista de Administración Pública, 58(2), 17-48.

Tramontana, E. (2010). The contribution of the Inter-American Human rights bodies to evolving International la won indigenous rights over lands and natural resources. International Journal on Minority and Group Rights, 17(2), 241-263.

Tsekos, M. E. (2002). Minority rights: the failure of International law to protect the Roma. Human Rights Brief, (9), 26-29. Recuperado de https://digitalcommons.wcl.american. edu/cgi/viewcontent.cgi?referer=https://scholar.google.com/\&httpsredir=1 \&article=14 $61 \&$ context=hrbrief

Unesco. (1990). Final Report and Recommendations of the International Meeting of Experts on further study of the concept of the rights of peoples. SHS-89/CONF.602/7.

Van Cott, D. L. (2000). The friendly liquidation of the past: the politics of diversity in Latin America. Pittsburgh, PA: University of Pittsburgh Press.

Weller, M. (2005). The rights of minorities. A commentary on the European Framework Convention for the Protection of National Minorities. Oxford, Inglaterra: Oxford University Press. 